\title{
Medición de volumen en pacientes en diálisis peritoneal: diferencias entre bioimpedancia espectroscópica y fórmula de Watson
}

\author{
Cristina Pérez Jiménez, Nemesio Manuel Martínez Martínez, Encarnación Bellón Pérez, Gonzalo Martínez \\ Fernández, Lourdes de la Vara Iniesta, Juan Pérez Martínez
}

\section{Servicio de Nefrología. Complejo Hospitalario Universitario. Albacete}

\section{Introducción y objetivos:}

Conocer el volumen corporal total (V) es fundamental en los pacientes en diálisis peritoneal (DP) dadas las implicaciones clínicas a las que se asocia. Habitualmente éste se calcula mediante la fórmula de Watson, aunque el empleo de bioimpedancia espectroscópica (BIS), dada su aplicabilidad, sencillez y la objetividad de sus resultados, cada vez está más generalizado en las Unidades de DP. En ocasiones, al medir V con ambos métodos surgen amplias diferencias, lo que genera un conflicto sobre cuál de los dos resultados aceptar como válido.

\section{Objetivo:}

Evaluar si surgen diferencias entre ambas formas de medir V en una Unidad de DP. Analizar qué factores clínicos frecuentemente presentes en los pacientes en DP se asocian a estas diferencias.

\section{Material y métodos:}

Estudio observacional realizado en 74 pacientes en DP. Medimos V empleando BIS (Vbis) y fórmula de Watson ( $\mathrm{Vw}$ ), obteniendo un total de 271 mediciones por ambos métodos, y comparamos si existen diferencias entre uno y otro volumen (Test T-Student). Calculamos la diferencia entre volúmenes en cada paciente y los clasificamos en dos grupos de acuerdo a si la diferencia entre volúmenes es $\geq 10 \%$ ó $<10 \%$ de Vbis. Hemos valorado una serie de parámetros en los pacientes analizados, clasificándolos según la pre- sencia o ausencia de cada factor: HTA, dislipemia, diabetes mellitus (DM), vasculopatía medida por ITB, cardiopatía isquémica, neuropatía isquémica, antiagregación o anticoagulación, sexo, edad ( $\geq 0<65$ años), variedad de DP, albúmina sérica ( $\geq 0<3.5 \mathrm{~g} / \mathrm{dl}$ ), obesidad ( $\mathrm{IMC} \geq 30 \mathrm{Kg} / \mathrm{m} 2$ ), E/I ratio ( $\geq 0<1$ ) y función renal residual $(F R R)(\geq 0<400 \mathrm{cc})$. Posteriormente valoramos si existe asociación entre las diferencias entre volúmenes y la presencia o ausencia de los parámetros clínicos descritos (Test Chi-Cuadrado).

\section{Resultados:}

Vw medio fue 2.15 litros mayor Vbis medio $(p<0.01)$. En el $58.67 \%$ de las mediciones, la diferencias entre volúmenes era $\geq 10 \%$ de Vbis. Aparecen diferencias significativas al comparar la presencia o ausencia de diferencia entre volúmenes y la presencia o no de $D M$ $(p=0.03)$, HTA $(p=0.036)$, hipoalbuminemia $(p<0.001)$, obesidad $(p=0.027), E / I$ ratio $>1(p<0.001)$ y diuresis residual $\geq 400 \mathrm{cc}(p=0.029)$.

\section{Conclusiones:}

Existen diferencias significativas en $\mathrm{V}$ de los pacientes de una Unidad de DP según sea calculado por fórmula de Watson u obtenido por BIS. La presencia de HTA, DM, hipoalbuminemia, obesidad, E/I ratio $>1$ y la FRR se asocia con la aparición de estas diferencias. Por tanto, los pacientes hipertensos, diabéticos, obesos, desnutridos y sin FRR se benefician de la medición de $\mathrm{V}$ por BIS. 